\title{
Efecto de la competición sobre la impulsividad del árbitro de fútbol amateur
}

\author{
Effect of the competition on amateur soccer referee impulsivity \\ José López-Aguilar ${ }^{1}$, Itziar Alonso-Arbiol ${ }^{2}$, Wanesa Onetti-Onetti ${ }^{3}$, Alfonso Castillo-Rodríguez ${ }^{1}$ \\ 1 Departamento de Educación Física y Deportiva. Universidad de Granada. España. \\ 2 Universidad del País Vasco UPV/EHU, Donostia-San Sebastián, España. \\ 3 Universidad Internacional de la Rioja, Logroño, España.
}

CORRESPONDENCIA:

Alfonso Castillo-Rodríguez

acastillo@ugr.es

Recepción: noviembre 2020• Aceptación: abril 2021
CÓMO CITAR EL ARTÍCULO:

López-Aguilar, J., Alonso-Arbiol, I., Onetti-Onetti, W., \& CastilloRodríguez, A. (2021). Efecto de la competición sobre la impulsividad del árbitro de fútbol amateur. Cultura, Ciencia y Deporte, 16(49), 519-528. http://dx.doi.org/10.12800/ccd.v16i49.1619

\section{Resumen}

El árbitro de fútbol (AF) se expone a situaciones estresantes durante la competición que pueden afectar a la toma de decisiones, pudiendo ser más o menos impulsiva durante dicha competición. Los objetivos de este estudio son analizar la fluctuación de la impulsividad del AF amateur entre un contexto basal y otro precompetitivo y, además, comparar la impulsividad del AF en función de la categoría, edad y experiencia. Participaron 29 árbitros españoles, todos hombres de 23.98 \pm 2.66 años; $73.64 \pm 7.15$ kg; y $7.8 \pm 2.6$ temporadas de experiencia de media. Se recogieron datos de la impulsividad mediante el cuestionario UPPS-P en 57 partidos de sus correspondientes categorías y posteriormente, entre 48 horas y 72 horas después se realizó de nuevo el cuestionario como toma basal. Este estudio tuvo lugar durante la temporada 2019-2020. Los resultados indicaron que los AF tienen mayores niveles de urgencia negativa, búsqueda de sensaciones e impulsividad total en estados precompetitivos que en basales $(p<.05)$. Asimismo, los AF con mayor categoría poseían menores niveles de urgencia negativa y positiva $(p<.05)$. Como conclusión, el AF amateur posee mayor impulsividad en estados previos a la competición y, además, aquellos con mayor categoría tienden a poseer menores niveles de impulsividad.

Palabras clave: personalidad, impulsividad, UPPS-P, fútbol, habilidades psicológicas.

\section{Abstract}

The soccer referee (SR) exposes themselves to stressful situations during the competition that may affect decision-making and may be more or less impulsive during the competition. The objectives of this study are to analyze the fluctuation of impulsivity of amateur AF between a basal context and a precompetitive one and, moreover, to compare the impulsivity of AF according to category, age and experience. Participants were 29 Spanish referees of $23.98 \pm 2.66$ years participated; $73.64 \pm 7.15 \mathrm{~kg}$; and $7.8 \pm 2.6$ seasons of experience on average. participated and data was collected from Impulsivity data were collected using the UPPS-P questionnaire in 57 matches of their corresponding categories and later, between 48 hours and 72 hours later, the questionnaire was carried out again as a basal intake. This study took place during the $2019-2020$ season. The results indicated that the referees have higher levels of negative urgency, sensation seeking and total impulsivity in precompetitive states than in basal states $(p<.05)$. Likewise, the SR with a higher category had lower levels of negative and positive urgency $(p<.05)$. As a conclusion, amateur SR has greater impulsivity in pre-competition states and, furthermore, those with a higher category tend to have lower levels of impulsivity.

Key words: personality, impulsivity, UPPS-P, football, psychological skills. 


\section{Introducción}

La práctica del fútbol es muy habitual entre la población, siendo el deporte más practicado en el mundo según Pérez-Gómez et al. (2020), contabilizándose más de 265 millones de practicantes (Malaguti et al., 2019). Más de 5 millones de árbitros de fútbol (AF) en todo el mundo desempeñan su labor de juez (FIFA, 2007; Malaguti et al., 2019). Los AF son deportistas con la función esencial de hacer que se desarrolle la competición oficial de manera correcta realizando tomas de decisiones de acuerdo al reglamento (Soriano et al., 2018), entendiendo este como las reglas de juego elaboradas por la International Football Association Board (IFAB, 2020).

Para ello, y como consecuencia de que el rendimiento físico de los jugadores es mayor (Rampini, 2007), tanto árbitros principales como árbitros asistentes deben tener una buena condición física que les permita recorrer largas distancias y cortas e intensas similares a las de los jugadores de fútbol (Rebolé et al., 2016; Di Salvo, 2007; Barros, 2007). Además de una buena condición física que permita al AF estar cerca de las jugadas, también es preciso poseer ciertas habilidades psicológicas, como la autoconfianza, concentración y motivación (Castillo-Rodríguez et al., 2020; Ramírez et al., 2006) y autoeficacia (Guillén \& Feltz, 2011; Guillén et al., 2019), entre otras, relacionadas con el rendimiento deportivo que, en un contexto del estrés permanente de la competición (Soriano et al., 2018), les permitan tomar decisiones ante situaciones extremas (público, posibles ascensos, descensos...) y, en consecuencia, lograr un arbitraje excelente (Garcés de lo Fayos \& Vives, 2003; Giske et al,. 2016; Weinberg \& Richardson, 1990). Estas habilidades psicológicas pueden desarrollarse si se trabajan (González-Oya \& Dosil, 2004, 2007; Guillén \& Feltz, 2011; Ramírez et al., 2006).

El estrés y el control del mismo al que están sometidos los AF es similar al que experimentan diversos deportistas, por ejemplo, tal y como se ha visto en comparación con el estrés soportado por un delantero (González-Campos et al. 2017; González-Oya, 2006) o un jugador de voleibol (Reyes \& Perez-Farinós, 2020; Noce, 1999), por ello, estos deportistas deben poseer un control del estrés óptimo, al igual que el AF, para que no les afecte en el rendimiento deportivo, pudiéndose traducir en lesiones o malas rachas de goles o puntos. El estrés puede desencadenar que el AF desarrolle una reacción subjetiva a niveles psicológicos y fisiológicos como consecuencia de percibir una situación o contexto como amenazante (González-Oya, 2006). Si este estrés no es controlado, y persiste en el tiempo, podrían producirse alteraciones de la percepción del entorno y la memoria además de a nivel físico/ corporal, temporal, cognitivo (Tornero-Aguilera et al., 2017) y fisiológico (González-Oya, 2006). A su vez, estas alteraciones podrían causar mayor impulsividad e imprecisión en la toma de decisiones del AF, debido a que podrían producir que no reuniera suficiente información del medio (Castillo-Rodríguez et al., 2018). En este sentido, el comportamiento del AF durante el partido es producto de los estados psicológicos previos (Kuroda et al., 2017). Por ello, la toma de decisiones y el rendimiento físico (en cuanto a distancias recorridas, desplazamientos a diferentes velocidades) y fisiológico (incremento o descenso de la temperatura corporal, frecuencia cardiaca durante el partido) del AF durante la competición parece estar relacionado con el estrés (Castillo-Rodríguez et al., 2020; Muñoz-Arjona \& Castillo-Rodríguez, 2020) y la impulsividad (Gaoua et al., 2017).

Los propios AF, en el estudio cualitativo de Lane et al., 2006, refirieron el tema del autocontrol/impulsividad como un elemento esencial para la correcta toma de decisión arbitral, siendo el autocontrol la capacidad de suprimir o alterar voluntariamente ciertas tendencias o impulsos de comportamiento para lograr objetivos más deseables a largo plazo (Samuel, 2015) y, en el caso del AF, para no precipitarse en una toma de decisiones. El autocontrol, componente relacionado con la impulsividad del AF, es una habilidad destacada por numerosos autores (Guillen \& Feltz, 2011; Samuel, 2015; Samuel et al. 2018, 2019; López \& Ordaz, 1992) para obtener un rendimiento óptimo como $\mathrm{AF}$ en la toma de decisiones.

La impulsividad es un constructo que presenta un carácter multidimensional (Arce \& Santiesteban, 2006; Evenden, 1999). Esta impulsividad está asociada a la búsqueda de sensaciones y la novedad (Cloninger et al., 1991; Zuckerman et al., 1993), persiguiendo pequeñas e inmediatas recompensas en vez de mayores recompensas que estén demoradas en el tiempo (Cherek \& Lane, 1999; Logue, 1988). Además, se encuentra asociada a la imposibilidad de evaluar riesgos que acompañan a determinados comportamientos o decisiones (Eysenck \& McGurk, 1980), o a la propensión de actuar sin pensar o antes de adquirir suficiente información para tomar una decisión correcta (Barrat, 1994), así como con la dificultad para manejar fuertes impulsos cuando se enfrentan a estados emocionales positivos o negativos (American Psychiatric Association, 2004; Whiteside et al., 2005).

Una forma de operacionalizar de manera válida y fiable es por medio del cuestionario UPPS-P, validado en población española por Cándido et al. (2012). Ade- 
más de proporcionar una puntuación global, también recoge la multidimensionalidad (con cinco dimensiones). La urgencia positiva (Urg+) y urgencia negativa (Urg-) son dimensiones referidas a la tendencia de experimentar fuertes reacciones ante posibles situaciones positivas o negativas, respectivamente; la falta de premeditación (PR) es la tendencia a no pensar las consecuencias de una acción determinada antes de realizarla; la falta de perseverancia (PE), considerada como la incapacidad de mantener la concentración en alguna tarea concreta que puede llegar a ser de larga duración, aburrida o que presente alguna dificultad para la persona; y búsqueda de sensaciones (SS), la cual está asociada a la tendencia de realizar actividades excitantes asociadas a conductas de riesgo.

La impulsividad en los AF, atendiendo a los diferentes aspectos arriba descritos, no ha sido objeto de estudio hasta la fecha, por lo que no existe evidencia científica al respecto. No obstante, ha sido evaluada en diversos deportistas como en jugadores profesionales de futsal, observando diferencias entre jugadores atacantes y defensores, siendo estos últimos quienes obtuvieron menores índices de impulsividad, donde además se constató la relevancia de hacer un examen más pormenorizado de las diferencias de impulsividad según las dimensiones (Castillo-Rodríguez et al., 2018).

El comportamiento defensivo orientado a la recuperación del balón (Castelão et al., 2014) exige niveles elevados de concentración (Silva et al., 2014) con el fin de no cometer errores que puedan suponer consecuencias negativas para el partido, evitando adoptar riesgos innecesarios a la hora de resolver una situación determinada. Teniendo en cuenta este razonamiento, los AF podrían tener un perfil similar al jugador defensivo, debido a la necesaria atención que deben mantener y elevada concentración durante la competición para hacer frente a las condiciones adversas o extremas comentadas anteriormente (Guillén \& Feltz, 2011).

Por otro lado, la experiencia del deportista en la competición, y en nuestro caso del AF, influye de manera positiva en el dominio de las habilidades psicológicas, como el estrés, así como en otros gremios como puede ser el ámbito militar, de la medicina, de la psicología y policía (Tornero-Aguilera \& Clemente-Suárez, 2018). Esta experiencia, basada en el ámbito militar, permite regular/modular las respuestas psicológicas y fisiológicas al estrés, el cual tiene una fuerte influencia en la percepción de la acción a nivel visual y motor (Tornero-Aguilera et al., 2017). Asimismo, y en cuanto al AF se refiere, Soriano et al. (2018) concluyen en su estudio que la relación experiencia/estrés percibido sugiere una relación negativa, encontrándonos así que los $\mathrm{AF}$ experimentados poseen menores niveles de estrés percibido $y$, en consecuencia, que los AF no experimentados obtendrán mayores niveles de estrés percibido durante la competición. Este hecho también lo indicó el estudio de González-Oya \& Dosil (2004) en $\mathrm{AF}$ gallegos, donde se encontró que los colegiados con mayor control del estrés son aquellos de más de 4 años de experiencia y de mayor categoría, mientras que los AF noveles, no experimentados y categoría inferior presentaban menor control del estrés. A similares conclusiones se llegó en un estudio realizado en Turquía, siendo aquellos AF que llevaban más de 15 años de experiencia los que mostraban un mayor control del estrés (Micoogullari et al., 2017). También debemos destacar que los AF de superior categoría tienden a obtener mayores niveles de autoeficacia, lo que provoca que mitiguen o vean reducidos sus niveles de estrés durante los partidos (Guillén, 2003), así como en general un mayor uso de estrategias de entrenamiento mental a niveles de entrenamiento físico similares (Giske et al., 2016).

En cuanto a la relación de la experiencia con la impulsividad del AF, no existe evidencia científica previa. No obstante, de acuerdo a los estudios de Mirzaei et al. (2013) y Stratton et al. (2004) se concluye que la experiencia permite inhibir respuestas o decisiones automáticas, encontrando mayor disciplina y responsabilidad en deportistas con mayor rendimiento. Además, los estados psicológicos previos del deportista influyen en el comportamiento de este (Kuroda, 2017) y por ello también en la toma de decisiones del AF. Por un lado, el estrés afecta más a AF noveles (Soriano et al., 2018; González-Oya \& Dosil 2004), los cuales tendrían una mayor activación a niveles psicofisiológicos que, si perdura en el tiempo, podría afectar a la percepción del colegiado (Tornero-Aguilera et al., 2017; Tornero-Aguilera \& Clemente-Suárez, 2018). Este hecho puede producir una tendencia del AF a tomar una decisión antes de obtener suficiente información del medio pudiendo no ser la acertada (Barrat, 1994).

Por tanto, este estudio tiene un doble objetivo. En primer lugar, analizar la fluctuación de la impulsividad del AF amateur entre un contexto basal y otro precompetitivo. Por otro lado, comparar la impulsividad del $\mathrm{AF}$ en función de la categoría, edad y experiencia. A continuación, planteamos las hipótesis:

H1: los AF tendrán mayores niveles de impulsividad en estados precompetitivos que en estados basales;

H2: los AF de menor categoría tendrán mayores niveles de impulsividad;

H3: los AF más experimentados y adultos tendrán menores niveles de impulsividad. 


\section{Método}

\section{Participantes}

En el presente estudio han participado voluntariamente veintinueve árbitros, hombres, pertenecientes al Comité Andaluz de Árbitros de Fútbol (España). De ellos, diecisiete pertenecen a la Categoría Provincial (en adelante Regional), cuatro pertenecen a la categoría División de Honor Sénior (en adelante Autonómica) y ocho a categoría Nacional (tres árbitros asistentes de $3^{\mathrm{a}}$ División y cinco AF $3^{\mathrm{a}}$ División). Todas las categorías eran masculinas.

La edad, peso, altura y experiencia en el arbitraje fueron de $23.98 \pm 2.66$ años; $73.64 \pm 7.15 \mathrm{~kg} ; 178.6 \pm$ $6 \mathrm{~cm}$ de altura; y $7.8 \pm 2.6$ temporadas de experiencia. Se recogieron los datos de las respuestas psicológicas del cuestionario de impulsividad en 57 partidos de sus correspondientes ligas a lo largo de la temporada 2019-2020. Se informó a los participantes de los procedimientos, objetivos, metodología, beneficios y posibles riesgos del estudio. Este estudio fue aprobado por el Comité de Ética de la Universidad de Granada (471/CEIH/2018).

\section{Instrumentos}

En primer lugar, se estableció un instrumento adhoc que permitía recoger los datos de cada árbitro correspondiente a la edad, talla, peso, experiencia en el arbitraje y experiencia en la categoría.

En segundo lugar, se procedió a la aplicación de la versión corta del cuestionario de impulsividad UPPSP (versión en español de Cándido et al., 2012), desarrollado en su versión original por Whiteside et al. (2005) y que contiene 20 ítems. El cuestionario diferencia cinco dimensiones de la impulsividad: urgencia positiva (Urg+; p.ej., "actúo de forma precipitada bajo emociones positivas"), urgencia negativa (Urg-; p.ej., "actúo de forma precipitada bajo emociones negativas"), PR (p.ej., "falta de consideración de las consecuencias que tendrán mis decisiones"), falta de perseverancia (PE; p.ej.; "tendencia a dejar de hacer tareas que me parecen aburridas o demandantes") y búsqueda de sensaciones (SS; p.ej.; "predisposición a realizar actividades novedosas"). Las propiedades psicométricas de la versión en español son adecuadas con los puntajes de confiabilidad de Cohen que varían entre .61 y .81 (Cándido et al., 2012). Sin embargo, en el presente estudio se obtuvieron coeficientes alfa de Cronbach comprendidos entre .751 y .886 en las variables en estado basal y entre .758 y .867 en estado precompetitivo (Tabla 1).

\section{Procedimiento}

Este es un estudio de corte longitudinal, inferencial de carácter descriptivo que tuvo lugar entre los meses de octubre de 2019 a marzo de 2020. En primer lugar, se localizó a los AF para explicarles los objetivos, metodología y protocolos de investigación. Además, se les facilitó el consentimiento informado y se les avisó de que cuando arbitraran partidos de su categoría contactaríamos con ellos para que realizaran los cuestionarios precompetitivos 60 minutos antes del mismo en las instalaciones deportivas. Y, posteriormente, tras más de 48 horas después de los partidos y no mas de 72 horas, volverían a realizar el cuestionario tomando esta toma como basal con el fin de ver si existen diferencias entre esta toma y los estados previos del partido con el fin de no estar influenciados por la siguiente competición. Como criterio de exclusión se estableció que los árbitros no hubiesen sufrido lesiones graves en los últimos 6 meses que les pudiera afectar en el desarrollo normal de los partidos.

\section{Análisis de datos}

Se utilizó el programa SPSS 25.0 para la realización del análisis estadístico. En primer lugar, para comprobar la normalidad de la muestra, se llevó a cabo la prueba Kolmogorov-Smirnov. Posteriormente se realizaron test comparativos y test de medidas repetidas ( $t$ de Student), tanto para muestras relacionadas como para muestras independientes. Para las variables no paramétricas se realizó el test de Wilcoxon para las medidas repetidas y $\mathrm{U}$ de Mann-Whitney para muestras independientes. Con el fin de comparar diferentes grupos/categorías se realizó la prueba ANOVA de una vía para variables paramétricas y $\mathrm{H}$ de Kruskal Wallis para variables no paramétricas y posteriormente se realizó un ajuste post-hoc de Bonferroni para hallar las diferencias entre los grupos. Se realizó una categorización de la muestra con el fin de obtener mayor información de esta (Ato et al., 2013). Así, en función de la experiencia dividimos la muestra en dos grupos uno de $\mathrm{AF}$ no experimentados ( $\leq 7$ temporadas) y otro de $\mathrm{AF}$ experimentados ( $\geq 8$ temporadas). En cuanto a la edad, hemos dividido al grupo en AF jóvenes (menor de 25 años) y AF adultos (mayor o igual a 25 años). El tamaño del efecto en el ANOVA se presenta mediante $\eta^{2}$ y se interpretó utilizando los siguientes criterios: efecto mínimo $(\eta 2 \leq .02)$, efecto moderado $\left(.02<\eta^{2}\right.$ $\leq .09)$ y efecto fuerte $\left(\eta^{2}>\right.$.09) (Lakens, 2013). En cuanto al tamaño del efecto para las pruebas $t$ de medidas repetidas y de muestras independientes, se interpretan con valores $d$ de Cohen; para la interpretación de 
este tamaño del efecto se utilizaron los siguientes criterios: efecto pequeño $(d<.20)$, efecto moderado $(.20 \leq$ $d<.80)$ y efecto grande $(d \geq .80)$ (O’ Donoghue, 2013).

\section{Resultados}

En la Tabla 1 se muestran los resultados de los test de normalidad y fiabilidad, respectivamente. Se observa que las dimensiones evaluadas muestran unos valores de fiabilidad medio-altos, excepto en la dimensión Urg- (en ambos contextos) y en PE y SS en el contexto basal y precompetitivo, respectivamente.

Tabla 1. Normalidad y fiabilidad ( $\alpha$ de Cronbach) de las dimensiones de impulsividad (test UPPS-P).

\begin{tabular}{ccccc}
\hline & \multicolumn{2}{c}{ Normalidad } & \multicolumn{2}{c}{ Fiabilidad } \\
\hline & Basal & Precompetitivo & Basal & Precompetitivo \\
\hline Urg- & .112 & .115 & .832 & .854 \\
Urg+ & .122 & .160 & .751 & .773 \\
PR & .162 & .161 & .816 & .839 \\
PE & .148 & .142 & .756 & .867 \\
SS & .114 & .119 & .886 & .758 \\
\hline
\end{tabular}

Urg-: Urgencia negativa; Urg+: urgencia positiva; PR: falta de premeditación; PE: falta de perseverancia; SS: búsqueda de sensaciones.

En la Tabla 2 podemos apreciar las diferencias entre las diferentes variables psicológicas estudiadas en cuanto a la categoría, habiendo diferenciado categoría Nacional, categoría Autonómica y categoría Regional, tanto en estados basales como precompetitivos. En cuanto a estados basales se aprecian diferencias signi- ficativas en la falta de perseverancia entre el grupo de AF Nacionales y el de AF Provinciales $(p<.05)$, sin encontrar otras diferencias significativas. Por otro lado, en cuanto a las diferencias entre los grupos en estados precompetitivos encontramos que los $\mathrm{AF}$ de categoría Nacional obtienen significativamente menores resultados en cuanto a Urg- $(p<.05)$ en comparación con los AF de categoría Autonómica y Regional. También se observa cómo los AF de categoría nacional tienen significativamente menores niveles de Urg+ que los AF provinciales.

En la Tabla 3 presentamos las medias, desviaciones estándar diferencias y tamaño del efecto entre las diferentes dimensiones de la impulsividad en estados basales y prepartido. Se muestra un aumento generalizado de las diferentes dimensiones siendo este aumento significativo en las variables de PR, SS e impulsividad total $(p<.05)$.

Tabla 3. Comparación de medias de las dimensiones de la impulsividad basales y pre-partido.

\begin{tabular}{cccccc}
\hline & $\begin{array}{c}\text { Basal } \\
(\mathbf{n}=\mathbf{5 7})\end{array}$ & $\begin{array}{c}\text { Precompetitivo } \\
(\mathbf{n}=\mathbf{5 7})\end{array}$ & $\mathbf{p}$ & $\mathbf{d}$ & \\
\hline Urg- & $10.96 \pm 2.72$ & $11.04 \pm 3.00$ & .827 & .03 & $\mathrm{P}$ \\
Urg+ & $9.55 \pm 2.53$ & $9.93 \pm 2.26$ & .182 & .16 & $\mathrm{P}$ \\
PR & $7.11 \pm 2.29$ & $7.77 \pm 2.83$ & .030 & .26 & $\mathrm{M}$ \\
PE & $7.36 \pm 2.14$ & $7.82 \pm 3.05$ & .187 & .17 & $\mathrm{P}$ \\
SS & $8.79 \pm 2.93$ & $9.70 \pm 2.55$ & .010 & .33 & $\mathrm{M}$ \\
TOT & $43.77 \pm 7.11$ & $46.25 \pm 7.46$ & .003 & .34 & $\mathrm{M}$ \\
\hline
\end{tabular}

Urg-: Urgencia negativa; Urg+: urgencia positiva; PR: falta de premeditación; PE: falta de perseverancia; SS: búsqueda de sensaciones. Para el tamaño del efecto (d) se utilizaron los siguientes criterios: efecto pequeño (P): $d<.20$; efecto moderado (M): $.20 \leq d<.80$; y efecto grande $(G): d \geq$ 80 (O’Donoghue, 2013).

Tabla 2. Comparación de medias de las dimensiones de la Impulsividad en tres categorías.

\begin{tabular}{|c|c|c|c|c|c|c|c|}
\hline & & $\begin{array}{l}\text { AF Categoría Nacional } \\
\qquad(\mathrm{n}=11)\end{array}$ & $\begin{array}{l}\text { AF Categoría Autonómica } \\
\qquad(\mathrm{n}=10)\end{array}$ & $\begin{array}{l}\text { AF Categoría Regional } \\
\qquad(\mathrm{n}=36)\end{array}$ & $p$ & $\eta^{2}$ & \\
\hline \multirow[t]{6}{*}{ Basal } & Urg- & $9.40 \pm 2.68$ & $11.80 \pm 1.55$ & $11.2 \pm 2.86$ & .106 & .081 & $\mathrm{M}$ \\
\hline & Urg+ & $8.60 \pm 3.17$ & $8.80 \pm 1.23$ & $10.0 \pm 2.54$ & .168 & .065 & $\mathrm{M}$ \\
\hline & PR & $7.90 \pm 2.60$ & $7.10 \pm 1.85$ & $6.9 \pm 2.32$ & .473 & .028 & $\mathrm{M}$ \\
\hline & PE & $7.40 \pm 2.17$ & $9.30 \pm 1.423$ & $6.8 \pm 2.012$ & .003 & .194 & $\mathrm{~F}$ \\
\hline & SS & $8.70 \pm 2.54$ & $7.80 \pm 3.33$ & $9.1 \pm 2.94$ & .479 & .027 & $\mathrm{M}$ \\
\hline & TOT & $42.00 \pm 6.78$ & $44.80 \pm 6.75$ & $44.0 \pm 7.39$ & .659 & .016 & $\mathrm{P}$ \\
\hline \multirow[t]{6}{*}{ Precompetitivo } & Urg- & $8.64 \pm 3.502 .3$ & $11.90 \pm 2.691$ & $11.4 \pm 2.781$ & .018 & .139 & $\mathrm{~F}$ \\
\hline & Urg+ & $7.82 \pm 2.233$ & $9.00 \pm 1.41$ & $10.7 \pm 2.141$ & .000 & .256 & $\mathrm{~F}$ \\
\hline & PR & $8.91 \pm 3.02$ & $8.10 \pm 1.60$ & $7.4 \pm 3.05$ & .321 & .041 & $\mathrm{M}$ \\
\hline & PE & $9.45 \pm 3.27$ & $8.60 \pm 2.32$ & $7.3 \pm 3.22$ & .111 & .078 & $\mathrm{M}$ \\
\hline & SS & $9.00 \pm 1.84$ & $9.40 \pm 3.03$ & $9.9 \pm 2.66$ & .585 & .020 & $\mathrm{M}$ \\
\hline & ТОТ & $43.82 \pm 5.53$ & $47.00 \pm 6.83$ & $46.7 \pm 8.04$ & .501 & .025 & $M$ \\
\hline
\end{tabular}

Urg-: Urgencia negativa; Urg+: urgencia positiva; PR: falta de premeditación; PE: falta de perseverancia; SS: búsqueda de sensaciones.

El tamaño del efecto $\left(\eta^{2}\right)$ y se interpretó utilizando los siguientes criterios: efecto mínimo (P) $\eta^{2} \leq .02$; efecto moderado (M): .02 $<\eta^{2} \leq .09$; y efecto fuerte (F): $\eta^{2}>.09$ (Lakens, 2013). 
Tabla 4. Comparación de medias de las dimensiones de la Impulsividad entre AF en función de la experiencia.

\begin{tabular}{|c|c|c|c|c|c|c|}
\hline & & $\begin{array}{l}\text { AF no experimentados } \\
\qquad(\mathrm{n}=28)\end{array}$ & $\begin{array}{l}\text { AF experimentados } \\
(\mathrm{n}=29)\end{array}$ & $p$ & & \\
\hline \multirow[t]{6}{*}{ Basal } & Urg- & $10.64 \pm 2.18$ & $11.29 \pm 3.17$ & .381 & .24 & $\mathrm{M}$ \\
\hline & Urg+ & $8.89 \pm 1.91$ & $10.21 \pm 2.91$ & .050 & .54 & $\mathrm{M}$ \\
\hline & PR & $7.61 \pm 2.01$ & $6.61 \pm 2.47$ & .102 & .44 & $\mathrm{M}$ \\
\hline & $\mathrm{PE}$ & $7.64 \pm 2.02$ & $7.07 \pm 2.24$ & .321 & .27 & $\mathrm{M}$ \\
\hline & SS & $8.00 \pm 2.83$ & $9.57 \pm 2.87$ & .044 & .55 & $\mathrm{M}$ \\
\hline & TOT & $42.79 \pm 6.85$ & $44.75 \pm 7.35$ & .305 & .28 & $\mathrm{M}$ \\
\hline \multirow[t]{6}{*}{ Precompetitivo } & Urg- & $10.64 \pm 2.45$ & $11.21 \pm 3.61$ & .495 & .18 & $\mathrm{P}$ \\
\hline & Urg+ & $9.50 \pm 2.08$ & $10.17 \pm 2.55$ & .281 & .29 & M \\
\hline & PR & $7.96 \pm 2.44$ & $7.72 \pm 3.25$ & .754 & .08 & $\mathrm{P}$ \\
\hline & $\mathrm{PE}$ & $7.93 \pm 2.85$ & $7.97 \pm 3.50$ & .965 & .01 & $\mathrm{P}$ \\
\hline & SS & $9.18 \pm 2.47$ & $10.07 \pm 2.65$ & .194 & .35 & M \\
\hline & TOT & $45.21 \pm 7.88$ & $47.14 \pm 6.91$ & .331 & .26 & $\mathrm{M}$ \\
\hline
\end{tabular}

Urg-: Urgencia negativa; Urg+: urgencia positiva; PR: falta de premeditación; PE: falta de perseverancia; SS: búsqueda de sensaciones.

Para el tamaño del efecto (d) se utilizaron los siguientes criterios: efecto pequeño (P): $d<.20$; efecto moderado (M): .20 $\leq d<.80$; y efecto grande (G): $d \geq .80$ (O'Donoghue, 2013). AF no experimentados: de 1 a 7 temporadas; AF experimentados: de 8 a 14 temporadas.

Tabla 5. Comparación de medias de las dimensiones de la Impulsividad en función de dos categorías de edad.

\begin{tabular}{|c|c|c|c|c|c|c|}
\hline & & $\begin{array}{c}\text { AF joven } \\
(n=26)\end{array}$ & $\begin{array}{c}\text { AF adulto } \\
(n=31)\end{array}$ & $p$ & & \\
\hline \multirow[t]{6}{*}{ Basal } & Urg- & $10.16 \pm 2.15$ & $11.61 \pm 2.97$ & .046 & .55 & $\mathrm{M}$ \\
\hline & Urg+ & $9.04 \pm 2.19$ & $9.97 \pm 2.74$ & .175 & .37 & $\mathrm{M}$ \\
\hline & PR & $7.76 \pm 2.03$ & $6.58 \pm 2.38$ & .054 & .53 & $\mathrm{M}$ \\
\hline & $\mathrm{PE}$ & $7.28 \pm 1.84$ & $7.42 \pm 2.38$ & .811 & .06 & $\mathrm{P}$ \\
\hline & SS & $8.44 \pm 2.18$ & $9.06 \pm 3.43$ & .412 & .21 & $\mathrm{M}$ \\
\hline & TOT & $42.68 \pm 5.81$ & $44.65 \pm 7.99$ & .292 & .28 & $\mathrm{M}$ \\
\hline \multirow[t]{6}{*}{ Precompetitivo } & Urg- & $9.88 \pm 3.18$ & $11.81 \pm 2.75$ & .018 & .65 & $\mathrm{M}$ \\
\hline & Urg+ & $9.04 \pm 2.01$ & $10.52 \pm 2.41$ & .016 & .66 & $\mathrm{M}$ \\
\hline & PR & $8.88 \pm 2.90$ & $6.97 \pm 2.55$ & .010 & .70 & $\mathrm{M}$ \\
\hline & $\mathrm{PE}$ & $8.50 \pm 3.55$ & $7.48 \pm 2.79$ & .231 & .32 & $\mathrm{M}$ \\
\hline & SS & $9.81 \pm 1.96$ & $9.48 \pm 3.02$ & .628 & .13 & $\mathrm{P}$ \\
\hline & TOT & $46.12 \pm 7.71$ & $46.26 \pm 7.26$ & .943 & .02 & $\mathrm{P}$ \\
\hline
\end{tabular}

Urg-: Urgencia negativa; Urg+: urgencia positiva; PR: falta de premeditación; PE: falta de perseverancia; SS: búsqueda de sensaciones.

Para el tamaño del efecto (d) se utilizaron los siguientes criterios: efecto pequeño (P): $d<.20$; efecto moderado (M): .20 $\leq d<.80 ;$ y efecto grande ( $G$ ): $d \geq .80$ (O'Donoghue, 2013). AF joven: < 25 años; AF adulto: $\geq 25$ años.

Las diferencias de las variables relacionadas con la impulsividad entre AF no experimentados y AF experimentados se pueden ver en la Tabla 4. En estados basales apreciamos incrementos significativos de las dimensiones de Urg+ y SS $(p<.05)$ en el grupo de AF experimentados, sin apreciar diferencias significativas en las demás variables basales, En cuanto a estados precompetitivos, no se han apreciado diferencias significativas entre los grupos en ninguna de las variables dependientes analizadas.

Las diferencias entre los grupos de edad se pueden observar en la Tabla 5. En cuanto a estados basales, apreciamos mayores niveles de Urg- en los AF adultos $(p<.05)$, sin encontrar diferencias significativas entre las demás variables. En cuanto a estados precompetitivos, se encuentran mayores niveles de Urg-y Urg+ en AF adultos $(p<.05)$ y menores niveles de PR $(p<.05)$ en comparación con los AF jóvenes.

\section{Discusión}

El presente estudio tenía un doble objetivo: a) analizar la fluctuación de la impulsividad del AF amateur entre contexto basal y precompetitivo, y b) comparar la impulsividad del AF en función de la categoría, 
edad y experiencia. Los resultados mostraron que los AF poseían mayor puntuación de las dimensiones de impulsividad en el contexto precompetitivo siendo significativo el incremento en las dimensiones de PR, SS e impulsividad total. Este hecho puede deberse al incremento del "arousal" o activación general a nivel fisiológico y psicológico (Gould \& Krane, 1992), plasmado en un incremento del estrés, que a niveles óptimos pueden ser beneficiosos para el rendimiento, ante una situación o contexto "amenazante", como podría ser el partido (González-Oya, 2006). En cuanto al incremento de PR, y definiéndola como la tendencia a no pensar las consecuencias de una acción determinada antes de realizarla (Cándido et al., 2012), pensamos que es causado por un incremento del estrés. Esto se produciría porque el AF debe tomar una decisión en décimas de segundo, percibiendo para ello la mayor información posible del medio y, además, valorando las posibles consecuencias. La demora en el tiempo de la toma de decisiones podría tener consecuencias negativas en el encuentro (agresiones, mayor presión de la grada, protestas de jugadores y técnicos...). En cuanto al incremento en la SS, y sabiendo que tiene una alta relación con la percepción y conductas de riesgo (Hansen \& Breivik, 2001), lo vemos lógico en estados precompetitivos, ya que el mero hecho de arbitrar un partido de futbol puede llegar a ser un riesgo para el colegiado, en el que puede sentirse cómodo en cierta manera tras haber adquirido unas habilidades psicológicas en el arbitraje (Dosil, 2004; Guillén \& Feltz, 2011). En cuanto al aumento de la impulsividad total es normal, ya que todas sus dimensiones se han incrementado, siendo las dos anteriores de manera significativa, y es por ello que finalmente se verifica la $\mathrm{H} 1$, en la que se confirma una mayor impulsividad por parte de los AF en el contexto precompetitivo.

Por otro lado, los AF de Categoría Nacional en estado precompetitivo mostraban menor impulsividad, concretamente en las dimensiones de Urg-, en comparación con las categorías Autonómicas y Regionales, y menor Urg+ en comparación con los Regionales. No poseemos antecedentes de estos resultados en otros manuscritos, ya que no existen, o no hemos podido tener acceso a ellos. No obstante, podemos razonar que los AF de categoría Nacional, al poseer menores niveles de Urg- y Urg+, les afectan menos las emociones que sienten tanto positivas como negativas a la hora de la toma de decisiones en comparación con categorías menores, debido a la mayor experiencia adquirida del manejo y control del estrés descrita en la literatura sobre AF (Soriano et al., 2018; González-Oya \& Dosil 2004). En cuanto a la PR se ven indicios de que, con una muestra mayor, podría haber diferencias significativas y podríamos relacionarla con la autoeficacia, definida como la percepción que el árbitro tiene de su propia capacidad para realizar tareas relacionadas con su función laboral (Guillén \& Feltz, 2011), ya que pensamos que los AF de mayor categoría poseen altos niveles de autoeficacia y, en consecuencia, seguridad en su toma de decisiones. Así, los AF de mayor categoría pueden poseer una tendencia a no valorar tanto las consecuencias de sus acciones, ya que pensarán que son las correctas. A pesar de que en AF nacionales contenían mayores valores no significativos de $\mathrm{PE}$, estos pueden ser debidos a que aquellos colegiados deben centrarse en más aspectos del juego, ya que según Cándido et al. (2012) la falta de perseverancia es considerada como la incapacidad de mantener la concentración en alguna tarea concreta que podría llegar a ser de larga duración. De esta manera, si el AF se centrase solo en una tarea o zona determinada podría perder la visión de otras acciones que suceden en el terreno de juego y que podrían ser claves para el correcto desarrollo del encuentro. Por último, los AF nacionales poseen menores niveles de SS de manera no significativa, lo que puede ser producido a raíz de que no quieren tomar decisiones precipitadas que puedan perjudicar a los equipos, ya que, a mayor categoría, mayor repercusión a nivel socioeconómico tendrán sus decisiones en los clubes (Ramírez et al., 2006). El poseer menores niveles de Urg- y SS, aunque no de manera significativa en esta última variable, podría relacionarse con el perfil de la impulsividad apreciados en los jugadores defensores de fútbol sala del artículo de Castillo-Rodríguez et al. (2018), pues el colegiado no quiere tomar decisiones arriesgadas que puedan perjudicar a alguno de los dos equipos y no se dejan influenciar por emociones negativas a la hora de tomar una decisión. Se podría afirmar de manera preliminar que se confirma la $\mathrm{H} 2$ de acuerdo a los resultados de significación estadística y tamaño del efecto, fuerte en dos dimensiones, y de tamaño del efecto medio en las restantes.

En cuanto a la experiencia de los AF, los resultados muestran que en estados basales los AF experimentados poseen mayores puntuaciones de urgencia positiva y búsqueda de sensaciones sin tener diferencias significativas en las otras dimensiones, aunque teniendo mayores puntuaciones en estados basales. En estados precompetitivos no se aprecian diferencias significativas entre grupos. Sí se aprecian mayores puntuaciones, por lo general, en los AF experimentados, sin ser estadísticamente significativas estas diferencias. Este hecho podría estar relacionado con la autoeficacia, si bien es cierto que solo en estados basales hay diferencias significativas en cuanto a Urg+ y SS podríamos pensar al tener mayor confianza en sus capacidades, 
y tras buenas tomas de decisiones, pueden llegar a ser más impulsivos por el hecho de poseer mayor experiencia y tener más seguridad en su toma de decisiones. Por lo tanto, debemos refutar la $\mathrm{H} 3$, pues los AF de futbol experimentados obtienen mayores puntuaciones de impulsividad de manera no significativa.

En cuanto a la comparativa entre la edad de los AF, hemos encontrado que a niveles basales los AF adultos tienen mayor Urg- que los AF jóvenes. Esto pudiera explicarse por otra serie de variables externas que tengan que ver con el número de estresores no relacionados con el arbitraje. Los dos grupos de edad se sitúan ambos en una franja de adultez joven. El grupo mayor de esta muestra (de 25 a 31 años) con mayor probabilidad pudiera encontrarse en situaciones laborales y/o familiares (hijos/as pequeños/as) que provoquen mayor irritabilidad y cuyos actos impulsivos tengan consecuencias mayores en sus entornos. En la literatura de los rasgos de personalidad, los estudios que analizan los cambios debido a la edad que implican disminución/aumento en los niveles de algunas variables establecen un punto de corte cercano a los 30 años (p.ej., McCrae et al., 1999; Srivastava et al., 2003).

Asimismo, en estados precompetitivos vemos que los AF adultos obtienen mayores puntuaciones de Urg-, Urg+ y menores puntuaciones PR comparando con los AF jóvenes. Estos datos podemos justificarlos de manera que la Urg- puede tener una relación con la motivación de los colegiados y la toma de decisiones ante situaciones emocionales negativas. $\mathrm{La} \mathrm{Urg}+$, como enunciamos anteriormente, puede estar relacionada con la autoeficacia porque, aunque los AF tengan mayores niveles de Urg+, toman decisiones porque confían en que van a ser acertadas. No obstante, y con relación a la Urg+, la PR es menor, por lo que también evalúan los riesgos de las decisiones y toman la máxima información de las situaciones del partido que ellos consideran necesaria y toman decisiones influenciados posiblemente por su autoeficacia. Tras analizar y discutir los datos obtenidos, podemos pensar que los AF jóvenes, a la hora de la toma de decisiones, no se dejan llevar por las emociones positivas o negativas, pudiendo ser, quizás, por no arriesgar a tomar malas decisiones, por falta de autoeficacia o simplemente al no tener tan desarrolladas unas habilidades psicológicas de manera óptima.

El conocimiento no solo de los rasgos de la impulsividad, sino también de otras variables psicológicas basándonos en el estudio de Castillo-Rodríguez et al. (2018) realizado con jugadores de fútbol sala y extrapolándolo al ámbito del arbitraje en fútbol, o el arbitraje en general, podría ser información muy valiosa para los delegados y en general para el CTA con el fin de rediseñar o realizar programas de intervención psicológica en $\mathrm{AF}$ con el fin de conocer y mejorar su rendimiento en cuanto a variables psicológicas se refiere. Sabiendo que son protagonistas de un deporte donde se requiere de un asesoramiento a nivel psicológico, estas son las demandas menos atendidas (Garcés de los Fayos \& Vives 2003), a pesar de que los propios árbitros (en un estudio con AF de primera y segunda división costarricense) mencionaron aspectos relacionados con la necesidad de controlarse ante situaciones inesperadas y/o que provocan inestabilidad (i.e. impulsividad), como el mayor de los defectos a trabajar para un buen desempeño arbitral (Villalobos et al., 2002). Estos programas, podrían beneficiar a los AF noveles, pues a menudo carecen de los recursos psicológicos necesarios (González-Oya \& Dosil, 2004). Diversos autores (Ramírez et al., 2006; González-Oya \& Dosil, 2007; Alonso-Arbiol et al., 2005) indican la importancia de estos programas de entrenamiento e intervención psicológica que les permita a todos los árbitros adquirir unos conocimientos y mecanismos para afrontar situaciones difíciles durante el arbitraje, pudiendo ser especialmente importante en árbitros jóvenes, los cuales, tras agresiones verbales o físicas, podrían desembocar en el abandono de la práctica del arbitraje (Alonso-Arbiol et al., 2005), pero también de importancia significativa en árbitros más experimentados, pues el trabajo de esas habilidades podrían traducirse en perfeccionamiento de su labor arbitral (Guillén, 2003; Fernández López, 1999, en Ramírez et al., 2006), plasmándose en ascensos de categoría. En cuanto a los programas psicológicos en AF destacamos el "Programa de Intervención en Habilidades Psicológicas en el Arbitraje” (PIHPA) llevado a cabo por Ramírez el al. (2006), el cual fue realizado de manera presencial en AF de $3^{\mathrm{a}}$ División de los comités territoriales valenciano y vasco y teniendo un alto grado de satisfacción general por parte de los árbitros. Este programa, según sus autores, podría llevarse a cabo en diferentes deportes y categorías, siempre y cuando sus contenidos se adaptaran específicamente a la población de árbitros en la que se pretende intervenir. En cuanto a la metodología a adoptar en el futuro que los propios autores, y en base a Garcés de los Fayos \& Vives (2003), proponen en sus conclusiones la formación mixta, presencial y a distancia (on-line) a lo largo de la temporada, junto con una preparación física optima, con el fin de adquirir y afianzar los conocimientos adquiridos. Este hecho de programas mixtos, unido a la necesidad apreciada por Soriano et al. (2018) de tratar en programas psicológicos los estresores de los árbitros no solo en el partido, sino fuera de ellos, podría producir una mejor relación entre los comités y los árbitros, produciendo una mejor experiencia y compromiso del colectivo arbitral. 
En cuanto a futuras vías de investigación sería interesante estudiar la relación entre la impulsividad y la autoeficacia por los datos obtenidos en el estudio, así como ampliar la investigación a otras disciplinas deportivas donde exista más de un árbitro con roles y capacidad de decisión similares (p.ej., baloncesto). Acerca de las limitaciones, la muestra se vio afectada en número debido a la pandemia del Covid-19, razón por la que algunas comparativas ofrecen un número de participantes por grupo reducido y que apunta a la conveniencia de realizar estudios en el futuro con un mayor número de AF para corroborar los resultados aquí observados.

\section{Conclusiones}

Los principales hallazgos del estudio muestran que el AF es más impulsivo en estados precompetitivos que en estados basales, posiblemente a causa del nivel de activación, y que ese cambio es susceptible de ser detectado empleando una metodología de autoinforme. Por otro lado, vemos que hay indicios, aunque no confirmación, de que los AF de mayor categoría y experiencia y edad son menos impulsivos. Finalmente existen indicios de que el AF de categoría Nacional tiende a ser más cauto a la hora de la toma de decisiones, posiblemente debido al impacto socioeconómico de su categoría.

\section{Agradecimientos y financiación}

Queremos manifestar nuestro agradecimiento al Comité Andaluz de Árbitros de Fútbol y a los árbitros que han participado en el presente estudio.

Este estudio ha sido financiado por el proyecto PPJIA2020.04, del programa Proyectos de Investigación Precompetitiva para Jóvenes Investigadores del Plan Propio 2020, de la Universidad de Granada.

\section{B I B LIOG R A FÍA}

Alonso-Arbiol, I., Falcó, F., López, M., Ordaz, B. \& Ramírez, A. (2005). Development of a questionnaire for the assessment of sources of stress in Spanish soccer referees. Ansiedad y Estrés, 11(2-3), 175-188.

American Psychiatric Association. (2004). Diagnostic and Statistical Manual of Mental Disorders Fourth Edition (Text Revision; DSM-IV- TR). Washington, DC: American Psychiatric Association.

Arce, E. \& Santisteban, C. (2006). Impulsividad: Una revisión [Impulsivity: A review]. Psicothema, 18, 213-220.

Ato, M., López-García, J. J., \& Benavente, A. (2013). Un sistema de clasificación de los diseños de investigación en psicología. Anales de Psicología, 29(3). doi:10.6018/analesps.29.3.178511

Barratt, E. S. (1994). Impulsiveness and aggression. In J. Monahan \& H. J. Steadman (Eds.), Violence and mental disorder: Developments in risk assessment (pp. 61-79). Chicago: University of Chicago Press.

Barros, R. M. L., Misuta, M. S., Menezes, R. P., Figueroa, P. J., Moura, F. A., Cunha, S. A., Anido, R. \& Leite, N. J. (2007). Analysis of the distances covered by first division Brazilian soccer players obtained with an automatic tracking method. Journal of Sports Science and Medicine, 6, 233-242.

Castelão, D., Garganta, J., Santos, R. \& Teoldo, I. (2014). Comparison of tactical behaviour and performance of youth soccer players in 3v3 and $5 \mathrm{v} 5$ small-sided games. International Journal of Performance Analysis in Sport, 14(3), 801-813. doi:10.1080/24 748668.2014.11868759

Castillo-Rodríguez, A., Madinabeitia, I., Castillo, A., Cárdenas, D. \& Alarcón, F. (2018). La impulsividad determina el rol desempeñado por los jugadores de futsal. Revista de Psicología del Deporte, 27, 181-188.

Castillo-Rodríguez, A., López-Aguilar, J. \& Alonso-Arbiol, I. (2020). Relación entre respuestas físico-fisiológicas y psicológicas en árbitros de fútbol amateur. Journal of Sport Psychology, 30(1), en imprenta.

Cherek, D. R. \& Lane, S. D. (1999). Laboratory and psychometric measurements of impulsivity among violent and nonviolent female parolees. Biological Psychiatry, 46, 273-280. doi:10.1016/S00063223(98)00309-6

Cloninger, C. R., Przybeck, T. R. \& Svrakic, D. M. (1991). The Tridimensional Personality Questionnaire: US normative data. Psychological Reports, 69, 1047-1057. doi:10.2466/pr0.1991.69.3.1047

Di Salvo, V., Baron, R., Tschan, H., Calderon Montero, F. J., Bachl, N., \& Pigozzi, F. (2007). Performance characteristics according to playing position in elite soccer. International Journal of Sports Medicine, 28, 222-227. doi:10.1055/s-2006-924294

Dorsch, K. \& Paskewich, D. (2007). Stressful experiences among six certification levels of ice hockey officials. Psychology of Sport and Exercise, 8, 585-593. doi:10.1016/j.psychsport.2006.06.003

Dosil, J. (2004). Psicología de la actividad física y del deporte. Madrid: McGraw-Hill.

Eysenck, S. B. \& McGurk, B. J. (1980). Impulsiveness and venturesomeness in a detention center populations. Psychological Reports, 47, 1299-1306. doi:10.2466/pr0.1980.47.3f.1299

Evenden, J. L. (1999). Varieties of impulsivity. Psychopharmacology, 146, 348-361. doi:10.1007/PL00005481

Fernández, J. A. \& López, M. (1999). Aspectos psicológicos de la actuación arbitral. Ponencia presentada en las Jornadas de Actualización Arbitral de la Federación Andaluza de Fútbol, Sevilla.

Gaoua, N., de Oliveira, R. F., \& Hunter, S. (2017). Perception, Action, and Cognition of Football Referees in Extreme Temperatures: Impact on Decision Performance. Frontiers in Psychology, 8, 1479. doi:10.3389/ fpsyg.2017.01479

Garcés de los Fayos, E. J. \& Vives, L. (2003). Formación en árbitros y jueces deportivos: Mejora de las competencias y habilidades psicológicas del árbitro. En F. Guillén (Dir.), Psicología del arbitraje y el juicio deportivo (pp. 161-186). Barcelona: Inde.

García-Mas, A. (2002). La psicología del fútbol. En J. Dosil (Ed.), El psicólogo del deporte: Asesoramiento e intervención (pp. 101-132). Madrid: Síntesis.

Gencay, S. (2009). Magnitude of psychological stress reported by soccer referees. Social Behavior and Personality, 37, 865-868. doi:10.2224/ sbp.2009.37.7.865

Giske, R., Hausen, T. \& Johansen, B. T. (2016). Training, mental preparation, and unmediated practice among soccer referees: An analysis of elite and sub-elite referees' reported practice. International Journal of Applied Sport Science, 28(1), 31-41.

González Campos, G., Valdivia-Moral, P., Cachón Zagalaz, J., Zurita Ortega, F., \& Romero, O. (2017). Influencia del control del estrés en el rendimiento deportivo: la autoconfianza, la ansiedad y la concentración en deportistas. Retos. Nuevas Tendencias en Educación Física, Deporte y Recreación, (32), 3-6. 
González-Oya, J. L. \& Dosil, J. (2004). Características psicológicas de los árbitros de fútbol de la Comunidad Autónoma Gallega. Cuadernos de Psicología del Deporte, 4(1-2), 53-66.

González-Oya, J. (2006). Psicología aplicada al árbitro de fútbol: Características psicológicas y su entrenamiento. Sevilla: Wanceulen.

González-Oya, J. \& Dosil, J. (2007). La Psicología del árbitro de fútbol. Noia: Toxosoutos.

Gould, D. \& Krane, V. (1992). The arousal athletic performance relationship: Current status and future directions. En T. Horn (Ed.), Advances in sport psychology (pp. 119-141). Champaign, Ill: Human Kinetics.

Guillén, F. (2003). Panorama actual en el estudio del arbitraje y el juicio deportivo desde una perspectiva psicológica, En F. Guillén (Dir.), Psicología del arbitraje y el juicio deportivo (pp. 7-24). Barcelona: Inde.

Guillén, F. \& Feltz, D. L. (2011). A conceptual model of referee efficacy. Frontiers in Psychology, 2, 1-5.

Guillén, F. Feltz, D., Gilson, T., \& Dithurbide, L. (2019). Analysis of the Psychometric Properties of the Spanish Version of Referee Self-Efficacy Scale (REFS). Revista de Psicología del Deporte, 28(1), 15-24.

Hansen, E. B. \& Breivik, G. (2001). Sensation seeking as a predictor of positive and negative risk behaviour among adolescents. Personality and individual differences, 30(4), 627-640. doi:10.1016/S01918869(00)00061-1

International Football Association Board (IFAB) (2020). Laws of the Game 2020/2021. Zurich: Fédération Internationale de Football Association. Recuperado de https://resources.fifa.com/image/upload/ ifab-laws-of-the-game-2020-21.pdf?cloudid=d6g1medsi8jrrd3e4imp \#: :text=FIFA\%20joined\%20The\%20IFAB\%20in,change\%20will\%20 benefit\%20the\%20game.\&text=For\%20every\%20proposed\%20 change\%2C\%20the,technology\%20can\%20benefit\%20the\%20game.

Kuroda, Y., Hudson, J., Thatcher, R. \& Legrand, F. (2017). Telic-paratelic Dominance and State Effects on Responses to Resistance and Endurance Exercise. Journal of Motivation, Emotion, and Personality, 6, 15-22. doi:10.12689/jmep.2017.603

Lakens, D. (2013). Calculating and reporting effect sizes to facilitate cumulative science: a practical primer for t-tests and ANOVAs. Frontier in Psychology, 4, 863. doi:10.3389/fpsyg.2013.00863

Lane, A. M., Nevill, A. M., Ahmad, N. S., \& Balmer, N. (2006). Soccer referee decision-making: 'Shall I blow the whistle?' Journal of Sports Science and Medicine, 5, 243-253.

Logue, A. W. (1988). Research on self-control: An integrated framework. Behavioral and Brain Sciences, 11, 665-709. doi:10.1017/S0140525X 00053978

López, M., \& Ordaz, B. (1992). Perfil estimado de la personalidad del árbitro de fútbol. Comunicación presentada en el I. Congreso de Psicología Profesional, Murcia.

McCrae, R. R., Costa, P. T. Jr., Lima, M. P., Ostendorf, F., Angeltiner, A., ... Piedmont, R. L. (1999). Age differences in personality across the adult life span: Parallels in five cultures. Developmental Psychology, 35 (2), 466-477. doi:10.1037/0012-1649.35.2.466

Micoogullari, B. O., Gumusdag, H., Odek, U., \& Beyaz, O. (2017). Comparative study of sport mental toughness between soccer officials. Universal Journal of Educational Research, 5, 1970-1976. doi:10.13189/ ujer.2017.051113

Mirzaei, A., Nikbakhsh, R. \& Sharififar, F. (2013). The relationship between personality traits and sport performance. European Journal of Experimental Biology, 3(3), 439-442.

Muñoz-Arjona, C., \& Castillo-Rodríguez, A. (2020). Attitude vs. Aptitude. Effect of psychological responses on soccer referees. In ternational Journal of Sport Psychology, 51(1), 69-80. doi:10.7352 IJSP.2020.51.069

Noce, F. (1999). Análise do estrés psíquico em atletas de voleibol de alto nivel:um estudio comparativo entre géneros. Disertación, mestrado. Escola de Educación Física da UFMG, Belo Horizonte, Brasil.

O'Donoghue, P. (2013). Statistics for sport and exercise studies: An introduction. (New York: Routledge).

Perales, J. C., Verdejo-García, A., Moya, M., Lozano, O. \& Pérez-García, M. (2009). Bright and dark sides of impulsivity: Performance of women with high and low trait impulsivity on neuropsychological tasks.
Journal of Clinical and Experimental Neuropsychology, 31(8), 927-944. doi:10.1080/13803390902758793

Pérez-Gómez, J., Adsuar, J. C., Alcaraz, P. E., \& Carlos-Vivas, J. (2020). Physical exercises for preventing injuries among adult male football players: A systematic review. Journal of Sport and Health Science. En imprenta. doi:10.1016/j.jshs.2020.11.003

Ramírez, A., Alonso-Arbiol, I., Falcó, F. y López, M. (2006). Programa de intervención psicológica con árbitros de fútbol. Revista de Psicología del Deporte, 15(2), 311-325

Rampinini, E., Coutts, A. J., Castagna, C., Sassi, R., \& Impellizzeri, F. M. (2007). Variation in top level soccer match performance. International Journal of Sports Medicine, 28, 1018-1024.

Rebolé, M., Castillo, D., Cámara, J., \& Yanci, J. (2016). Relación entre la capacidad cardiovascular y la capacidad de esprints repetidos en árbitros de fútbol de alto nivel. Revista Iberoamericana de Ciencias de la Actividad Física y el Deporte, 5(3), 49-64. doi:10.24310/riccafd.2016. v5i3.6141

Reyes Gómez, G., \& Pérez-Farinós, N. (2020). Influencia del estrés en el origen de lesiones en voleibol de competición. Revista Iberoamericana De Ciencias De La Actividad Física Y El Deporte, 9(3), 131-142. doi:10.24310/riccafd.2020.v9i3.10512

Samuel, R. D. (2015). A Psychological Preparation Framework for Elite Soccer Referees: A Practitioner's Perspective. Journal of Sport Psychology in Action, 6(3), 170-187. doi:10.1080/21520704.2015.1065938

Samuel, R. D., Englert, C., Zhang, Q., \& Basevitch, I. (2018). Hi ref, are you in control? Self-control, ego-depletion, and performance in soccer referees. Psychology of Sport and Exercise, 38, 167-175. doi:10.1016/j. psychsport.2018.06.009

Samulski, D. M. \& Noce, F. (2003). Estrés psicológico en árbitros de deportes colectivos. En F. Guillén (Dir.), Psicología del arbitraje y el juicio deportivo (pp. 109-131). Barcelona: INDE.

Silva, B., Garganta, J., Santos, R. \& Teoldo, I. (2014). Comparing tactical behaviour of soccer players in 3 vs. 3 and 6 vs. 6 small-sided games. Journal of Human Kinetics, 41(1), 191-202. doi:10.2478/hukin-2014-0047

Soriano Gillué, G., Ramis Laloux, Y., Torregrossa Álvarez, M., \& Cruz i Feliu, J. (2018). Sources of Stress Inside and Outside the Match in Football Referees. Apunts. Educación Física y Deportes, 132, 22-31. doi:10.5672/apunts.2014-0983.es.(2018/2).132.02

Srivastava, S. J., John, O. P., Gosling, S. D., \& Potter, J. (2003). Development of personality in early middle adulthood: Set like plaster or persistent change? Journal of Personality and Social Psychology, 84 (5), 1041-1053. doi:10.1037/0022-3514.84.5.1041

Stratton, G., Reilly, T., Richardson, D. \& Williams, A. M. (2004). Youth soccer: From science to performance. London: Routledge.

Tornero-Aguilera, J. F., Robles-Pérez, J. J. \& Clemente-Suárez, V. (2017). Effect of combat stress in the psychophysiological response of elite and non-elite soldiers. The Journal of Medical Systems, 41(6), 100. doi:10.1007/s10916-017-0748-x

Tornero-Aguilera, J. F., \& Clemente-Suárez, V. J. (2018). Effect of experience, equipment and fire actions in psychophysiological response and memory of soldiers in actual underground operations. International Journal of Psychophysiology, 128, 40-46. doi:10.1016/j.ijpsycho.2018.03.009

Villalobos, D., Ugarte, O. \& Guillén, F. (2002). Situación actual y expectativas del árbitro de fútbol profesional. Kinesis, 33, 13-20.

Voight, M. (2009). Sources of stress and coping strategies of US soccer officials. Stress and Health, 25, 91-101. doi:10.1002/smi.1231

Weinberg, R. S. \& Richardson, P. A. (1990). Psychology of officiating. Champaign, IL: Leisure Press.

Whiteside, S. P., Lynam, D. R., Miller, J. D. \& Reynolds, S. K. (2005) Validation of the UPPS impulsive behaviour scale: A four-factor model of impulsivity. European Journal of Personality, 19, 559-574. doi:10.1002/per.556

Zuckerman, M., Kuhlman, D. M., Joireman, J., Teta, P. \& Kraft, M. (1993). A comparison of three structural models for personality: The Big Three, the Big Five, and the Alternative Five. Journal of Personality and Social Psychology, 65, 757-768. doi:10.1037/0022-3514.65.4.757 